\title{
THEORETICAL PROCESSES IN STAR FORMATION
}

L. Mestel

Astronomy Centre, University of Sussex

\section{INTRODUCTION: FRAGMENTATION VERSUS DISSIPATION}

To bring out the basic problem of star formation, consider a nonrotating cosmical gas cloud which is free to contract and so to convert most of its gravitational energy into kinetic energy of mass motion. Fluid dynamical systems in general tend to dissipate their kinetic energy, and a diffuse gas cloud easily radiates excess heat. If the cloud did not break up into fragments, then the steady conversion of gravitational energy into radiation would lead ultimately in to the formation of a compact massive body - perhaps a quasar - but not of a star cluster, which is maintained at a comparatively low mean density by random stellar kinetic energy. Thus the transition from a gravitationally-bound cloud of gas and dust into a bound star cluster - or a fortiori into an expanding $0-B$ association - requires that gravitational energy released during collapse be conserved as the random macroscopic kinetic energy. It is very likely that star formation is often triggered by processes that are essentially dissipative, such as shocks following passage of the spiral wave, or cloud-cloud collisions; however, it is equally important to emphasise that we require at some stage a cut-off in kinetic energy dissipation. And indeed, once a cloud has been able to fragment into a group of self-gravitating blobs, each of small geometrical cross-section, the chance of inelastic collisions between blobs is sharply reduced.

The radius of the ultimate cluster is closely related to the amount of energy conserved during collapse and break-up. As an extreme case, suppose a cool, roughly spherical cloud of mass $M$, initially at rest at radius $R_{\rho}$, collapses and fragments completely and with negligible energy dissipation into a cluster of proto-stars. The random kinetic energy $T$ that maintains the cluster at its ultimate radius $R$ is then determined by the virial theorem $T_{r}=-V / 2$, where $V \simeq-G^{2} / R$ is the gravitational potential energy at ${ }^{r}$ radius $R$; combined with the conservation of energy $T_{r}+V=V_{0}$, this yields $R=R_{o} / 2$. As the density of a typical open cluster ${ }^{\circ}$ is in fact similar to that of a molecular cloud, it is clearly desirable that the first fragmentation should occur while 
the cloud is still of moderate density.

There will be a similar competition between fragmentation and dissipation when the cloud has a realistic angular momentum. In particular, it can be argued that what determines whether a proto-galaxy becomes disk-like or elliptical is the epoch at which energy dissipation is cut off. This in turn is related to the overall spin $\Omega$ of the protogalactic cloud, in that if $\Omega^{2} R^{3} / G M$ is close to unity, not only will the cloud as a whole be unable to contract in two dimensions, but any subcondensations trying to form will have large geometrical cross-sections, so that dissipative collisions remain probable until the cloud has substantially flattened along the rotation axis (cf. Section 4).

A non-rotating, non-magnetic gas cloud can in fact begin to contract only if its self-gravitation can overcome its internal pressure. A cool, dense, roughly spherical cloud at temperature $T$, with mean density $\rho$ and mean molecular weight $\mu$, immersed in a hot, low-density intercloud medium will collapse if its mass $M$ exceeds a critical mass $\mathrm{M}_{\mathrm{J}} \simeq 2\left[(\mathrm{QT} / \mu)^{3} / \mathrm{G}^{3} \rho\right]^{\frac{1}{2}}$ (Ebert 1955; Bonnor 1956; McCrea 1957). This "Jeans mass" is close to that of a sphere of density $\rho$ and radius equal to the "Jeans length" that emerges from the classical but non-rigorous treatment of gravitational instability. If we parametrize the $(\bar{\gamma}-\bar{s})$ then $\mathrm{M}_{\mathrm{J}} \propto \rho(3 \bar{\gamma}-4)$; hence if $\bar{\gamma}<4 / 3, \mathrm{M}_{\mathrm{J}}$ decreases as $\rho$ increases. In a diffuse HI or molecular cloud, compressional heating is negligible, and the balance between heating and cooling processes implies that $\bar{\gamma}<1$. In a hypothetical primeval gas cloud of pure hydrogen and helium, $T$ remains close to $10^{4} \mathrm{O}_{\mathrm{K}}$, and $\bar{\gamma} \simeq 1$. In either case, $\mathrm{M}_{\mathrm{J}}$ will in fact decrease as the cloud collapses. This is the basis of the simplest fragmentation model (Hoyle 1953; Mestel and Spitzer 1956; Gaustad 1963). If $M$ is only a little greater than the initial value of $M_{J}$, the cloud will begin by converting nearly all the gravitational energy released into thermal energy, which is immediately radiated away; and likewise the cloud cannot fragment, since $M \simeq M_{J}$. At later epochs the cloud goes over into nearly pressure-free collapse, with most of the further gravitational energy released becoming macroscopic kinetic energy of inflow. At the same time, since now $M \gg M_{J}$, the cloud can break up into a number of fragments, each of mass $M^{\prime} \simeq M_{J}$, and each exerting enough self-gravitation to withstand its internal pressure; and the kinetic energy of collapse is available to be converted via elastic encounters into random kinetic energy. Further, as each fragment itself collapses under its self-gravitation, then as long as $\bar{\gamma}$ stays well below 4/3,by the same argument sub-fragments can separate out. The hierarchy would terminate at densities sufficiently high for heat of compression to be effectively trapped rather than radiated away, so that $\bar{\gamma}$ becomes closer to $5 / 3$ rather than 1 .

The dynamics of this type of fragmentation differs from Jeans-type gravitational instability, in that we demand not just that a density fluctuation in a medium at rest should amplify when its mass exceeds $M_{J}$, but that it separate out from a medium that is itself in a state of 
overall collapse. Analytical studies of both the linear and postlinear domains (Hunter 1962, 1964; Lynden-Bell 1973) show that Hoyle's intuitive transition from "can fragment" to "will fragment" is very plausible. The danger that fragments may dissipate their energy in collisions and so coalesce (Layzer 1963) is probably only serious if the overall contraction of the cloud is strictly spherical - a highly improbable case (Lin, Mestel and Shu 1965).

In fact, when a realistic angular momentum is introduced it is no longer clear that Hoyle-Hunter fragmentation is relevant to the star formation problem (cf. Section 4). However, the idea of opacitylimitation to systematic break-up reappears in one form or another in most current discussions: we return to it in Section 7 . But first we re-discuss the dynamical problem, with more realistic physical conditions.

\section{GRAVITATIONAL COLLAPSE: THE ROLE OF THE GALACTIC MAGNETIC FIELD}

Consider now a cool, roughly spherical cloud, of mass greater than $\mathrm{M}_{J}$, but threaded by the lines of the local galactic magnetic field $\mathrm{B}$. As the cloud collapses, the frozen-in magnetic field is distorted and begins to exert forces opposing gravity. Intuitively one expects that if the field is not to be able to prevent collapse, then the mass must exceed a new critical value given approximately by replacing $R T / \mu$ by

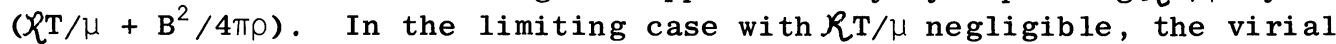
theorem (Chandrasekhar and Fermi 1953; Mestel and Spitzer 1956; Mestel $1965 ;$ Strittmatter 1966) does indeed yield a critical mass $M_{c} \propto$ $\left(\mathrm{B} / \rho^{2 / 3}\right)^{3} / \mathrm{G}^{3 / 2}$ where $\mathrm{B}$ and $\rho$ are simultaneous values in a roughly spherical cloud. However, as a cold spherical cloud will in fact flatten along the field, it is best to write this at once in terms of the magnetic $f l u x F=\pi B R^{2}$ through the equator. The mass-flux relation $\mathrm{GM}^{2}=5 \mathrm{kF}^{2} / 9 \pi^{2}$ fixes the minimum mass required to enforce gravitational collapse of a cloud containing a large-scale field with flux $F$ : the factor $k$ varies from 1 to $\simeq 1 / 4$ as the cloud flattens from a sphere into a highly oblate spheroid (Strittmatter 1966). Note that the density and the length-scales both along and across the field have disappeared.

At this point we digress and emphasise that quite generally the relation between $B$ and $\rho$ - with strict flux-freezing assumed - depends critically on the kinematics and so also on the dynamics of the gas flow. The erroneous idea is still abroad that flux-freezing necessarily implies the relation $B \propto \rho^{2 / 3}$, whereas in fact this holds only if the gas motion is isotropic. This is clearly correct during the contraction of a slowly rotating star, for which linear dimensions both along and across the field change by the same scale-factor $\lambda$ : flux and massconservation then yield respectively $B \propto 1 / \lambda^{2}$ and $\rho \propto 1 / \lambda^{3}$, so that $B \propto \rho^{2 / 3}$. This is the formula from which one can deduce an internal magnetic field within a white dwarf or a pulsar in terms of a corresponding field within the core of an evolving main sequence star. By contrast, the gas in a cool spherical cloud forming e.g. by thermal instability must flow preferentially along the field because of the anisotropy of the Lorentz force $j \times \mathcal{R}^{\mathrm{c}}$ : the contraction factor 
$\lambda_{\perp}$ across the field is less than $\lambda_{i l}$, and $B / \rho^{2 / 3}$ decreases. If the gas flow is initiated by the passage of the spiral shock wave, the $B-\rho$ relation will depend on the mutual orientation of the shock and the field. A shock front aligned across the field will cause flow down the field, so that $B$ stays constant while $\rho$ increases; whereas a shock parallel to the field will cause both $B$ and $\rho$ to increase like $1 / \lambda_{\perp}{ }^{2}$, so that $B \propto \rho$; and a shock inclined to the field will cause refraction of the lines of force. The Parker Rayleigh-Taylor instability yields flow down the field, so that $B / \rho^{2 / 3}$ will again decrease.

The situation is summed up succinctly by Vrba and the Stroms (1976): "The shape of a cloud and the field structure bear witness to the cause of collapse". As a further example, consider a cool cloud which forms as a highly prolate spheroid about the field direction. Self-gravitation always acts so as to increase eccentricity (Lin, Mestel and Shu 1965); just as an oblate spheroid tries to flatten, so a prolate spheroid tries to become cylindrical. Howe ve $r$, the consequent compression of the field yields a magnetic pressure $B^{2} / 8 \pi \propto \rho^{2}$, so that the spontaneous increase in eccentricity is now checked (cf. McCrea 1957). The collapse is thus more-or-less isotropic, with $\mathrm{B} / \mathrm{\rho}^{2 / 3}$ consequently staying constant

(Mestel 1965). However, this depends on special initial conditions: flattening and anisotropic collapse is more likely.

Note that a "uniform" field - i.e. one with a scale much larger than the cloud dimensions - exerts negligible force, and so does not interfere with the initial collapse. Clearly, if the mass $M<M_{C}(F)$, the cloud must try and reach a state of magneto-thermo-gravitational equilibrium, essentially with gravity balanced by thermal pressure along the field, and by the magnetic pressure gradient and curvature force across the field (Mestel 1965; Strittmatter 1966). A very rough estimate for the radius across the field of such a model is $R=$ $R_{0}\left(1-\left(M / M_{C}\right)^{2}\right)$, where $R_{\text {is }}$ is the radius from which the collapse across the uniform field began. The dimension along the field is essentially the thermal Jeans length. More recently, these approximate virial theorem treatments have been replaced by detailed accurate models of cool, isothermal gas clouds, immersed in a hot inter-cloud medium, and threaded by a magnetic field which becomes uniform at infinity (Parker, D.A. 1973, 1974; Mouschovias $1976 \mathrm{a}, \mathrm{b})$. Continuity of thermal pressure at the cloud surface ensures that the external density is low, so that only the cloud's self-gravitation is important; and as the tangential as well as the normal components of $\mathbb{R}$ are continuous, there are no surface currents and consequent unbalanced surface stresses.

Mouschovias's technique ensures the invariance of an initially prescribed distribution of mass within flux-tubes, as required by fluxfreezing: one can thus follow the evolution of a given cloud through a series of equilibria as the temperature falls; or compare the parameters of clouds of different mass, all forming from the same initial state. Such models can be plausibly used to describe clouds in which a locally enhanced field, as inferred from the Zeeman effect on the $21 \mathrm{~cm}$ line, is associated with a high density - due both to flow down the field and simultaneous lateral motion of gas and field-lines - and with a high 
cloud mass, exerting sufficient gravitation to balance the lateral magnetic forces (Mestel 1969; Verschuur 1969). However, it should be already clear that interpretation of these observations in terms of the "law" $B \propto \rho^{2 / 3}$ - even if it could be assumed that all clouds formed from regions with the same distribution of magnetic flux with respect to mass - is highly implausible; and Mouschovias argues reasonably that the observations are equally consistent with a lower index, as suggested by theory. After equilibrium models have been constructed, there remains the question of their stability, especially against nonaxisymmetric perturbations. A beginning to this formidable problem has been made by Garlick (1976) who has extended the hydromagnetic energy principle to non-rotating clouds with non-zero field at infinity.

Although so far no definite case of instability has yet been found, there are hints that instability will set in at least for some highly flattened models. It is surmised that rotation will encourage the spontaneous transformation into a non-axisymmetric structure, as in the MaclaurinJacobi problem.

\section{MAGNETIC FRAGMENTATION}

We now suppose that $M \geqslant M_{C}(F)$ so that the cloud will contract indefinitely (as long as centrifugal forces do not interfere). If the contraction were isotropic, then sub-condensations could not form at any $\mathrm{stage;}$ for a blob of mass $M^{\prime}=\lambda^{3} M(\lambda<1)$ would contain flux $F^{\prime}=\lambda^{2} F$, so that $G_{M}{ }^{2} / F^{\prime 2}=\lambda^{2} G^{2} / F^{2}$ would be sub-critical; or equivalently $B / \rho^{2 / 3}$ would stay, çonstant. But if the cloud spontaneously flattens along the field, $B / \rho^{2 / 3}$ decreases and smaller masses can separate out. If a cool spherical cloud of initial density $\rho$ and radius $\underline{R}$ has reached a thickness $\bar{z}$ and density $\bar{\rho}=\rho \underline{R} / \bar{z}$, then a blob of radius $\bar{z}$ at the cloud centre, containing flux $F^{\prime}=\pi \bar{z}^{2}=(\bar{z} / R)^{2} F$, has mass $M^{\prime}=4 \pi \rho \bar{z}^{3} / 3=M(\bar{z} / R)^{2}$, so that now $\mathrm{GM}^{\prime 2} / \mathrm{F}^{\prime 2}=\mathrm{GM}^{2} / \mathrm{F}^{2}$. Because of the flattening, sufficient mass has agglomerated by flow down the field to ensure that a quasi-spherical blob exerts enough self-gravitation to dominate over the forces exerted by a field of flux F' (Mestel 1965).

The argument so far ignores thermal pressure - we implicitly assume that the Jeans length is small compared with the scale of the cloud. It is well known (McCrea 1957) that isothermal, one-dimensional collapse is limited, since the pressure gradient $\simeq a^{2} \bar{\rho} / \bar{z}$ increases like $1 / \bar{z}^{2}$, while the z-component of the gravitational force density is a disk $\simeq G\left(\bar{\rho} \bar{z}^{3}\right) \bar{\rho} / \bar{z}^{2} \propto 1 / \bar{z}$. The minimum mass that can form, following the flattening of a magnetic cloud into a state of hydrostatic support along the field, will therefore be somewhat greater than $M(\bar{z} / R)^{2}$ - perhaps by an order of magnitude (Mestel 1965). However, the important points are: (1) fragmentation of an isothermal magnetic cloud can occur following preferential flow along the field, and the blobs that can form have masses determined by the ratio $(\bar{z} / R)$; (2) subsequent gravitational contraction across the field will reduce $\bar{z} / R$ still further, so allowing further break-up; (3) the magnetic flux does not appear explicitly in the mass of the fragments forming at each stage; (4) as the fragments are all "strongly magnetic", in the sense that the magnetic and gravi- 
tational energies are comparable, the masses forming at each density are certainly greater than those in the non-magnetic problem; (5) if the field is effectively frozen-in all the way to the opaque, pre-main sequence phase, it is again opacity effects that will fix the minimum stellar masses (cf. Section 7 ); (6) again we require conservation of a substantial fraction of the energy released by contraction as random kinetic energy of the fragments.

Tentative support for the picture of star formation following flow down the field-lines comes from the work of Vrba (1976) and Vrba and the Stroms (1976), summarised in the Symposium proceedings.

The time has now come for a computer attack on the formidable problem of the dynamics of collapse of a self-gravitating magnetic gas cloud, analogous to Mouschovias' studies of equilibrium mode1s. Earlier studies (e.g. Mestel 1966; Mestel and Strittmatter 1967) have necessarily been partly analytical and partly intuitive. However, some qualitative features, due essentially to the severity of the flux-freezing constraint, are likely to persist in a future detailed treatment. In any problem of gravitational collapse, the re is a strong tendency for the denser inner parts to run ahead of the outer. A frozen-in field will acquire a correspondingly distorted structure: the more-or-less uniform field within the centre will be linked with the background galactic field by nearly radial field-lines, which consequently exert strong pinching forces towards the equator. The outer parts of the cloud tend to be held up by the tensions of the distorted field-lines, a point implicit in the earlier studies and recently emphasized by Mouschovias $(1976 \mathrm{~b}, \mathrm{c})$. This structure forces re-appraisal of the strict flux-freezing assumption. In a lightly ionized gas the field moves with the ionized component via electromagnetic induction (except for Ohmic effects). The neutral gas moves under the non-electromagnetic forces, including the frictional force due to ionneutral particle collisions; the ionized component ( $p$ lus the field) moves under the joint action of the magnetic force and the same frictional force with reversed sign. The strong magnetic pinching forces at the equator begin by compressing both ionized and neutral components; however, the neutral gas can diffuse out of the equatorial zone under its partial pressure, leaving just the ionized gas to withstand the magnetic pressures. The strongly compressed ionized gas will then largely recombine into neutral atoms, which will again drift out of the pinched zone. This runaway process leads to the mutual separation of oppositely directed fieldlines becoming so small that Ohmic diffusion occurs - perhaps violently, as in a solar flare - leading to a steady detachment of the field in the cloud centre from the local galactic field (Mestel and Paris 1976).

This change in the topology of the field at some stage in the contraction of a cloud will clearly reduce the angular momentum transport by Alfvén waves travelling along the field-lines extending to infinity (cf. Section 4). However, a new point to stress is that a field which has acquired o-type neutral points is dynamically unstable against modes analogous to the familiar pinch instabilities. The problem has been discussed by Wright (1973) and Markey and Tayler (1973) in the context of 
magnetic star theory. In an opaque body such as a star, dynamicallydriven motions will be nearly adiabatic, so that the unstable modes are restricted by the condition that motions be nearly perpendicular to the gravitational field. But in a diffuse gas cloud a dynamical time-scale is sure to be much longer than the temperature equilibration time; motions in any direction will be nearly isothermal, and the adiabatic stabilization term in the energy integral vanishes. It is difficult to follow the non-linear development of these instabilities, but it is at least plausible that the field rapidly tangles itself sufficiently for Ohmic destruction of flux to occur. If this is correct, then as soon as the cloud field detaches from the background field, instabilities occur which tend to restore the field to an undetached topology. The process implies a reduction in the flux threading the cloud, essentially in an Alfvén time-scale, which in a strongly magnetic cloud is comparable with the free-fall time.

Rough estimates suggest that the detachment time is shorter than the free-fall time once the cloud has shrunk by a factor $\simeq 10$, so that subsequently the cloud will tend to be magnetically isolated from the background. However, if the cloud is not in free-fall, but is held up e.g. by centrifugal forces (cf. Section 4), then this dynamical flux destruction via instabilities may allow some magnetic contact between cloud and background to persist longer. The point to note now is that in a strongly magnetic cloud or fragment, hydromagnetic instabilities can lead to dynamical flux destruction, but in a time that is comparable with the free-fall time. Such flux loss is neither "fast", i.e. occurring in a time shorter than the free-fall time, and so rendering the magnetic forces weak compared with the gravitational; nor "slow", implying effective flux-freezing, as has been assumed so far. It may enable a cloud or fragment to lose any embarrassing excess flux, so perhaps helping further fragmentation (cf. Section 4 below), without simultaneously implying that the field becomes dynamically insignificant.

\section{ROTATING MAGNETIC CLOUDS}

The whole problem of collapse and fragmentation becomes more complicated when we give the cloud a realistic angular momentum, derived either from the galactic rotation or from the interstellar turbulence. To illustrate this, consider the idealization of a cloud of mass $M$ with a uniform angular velocity $\Omega$. If the angular momentum $H$ is strictly conserved - the analogue of $f\} y x$ conservation - then $\Omega$ increases under isotropic contraction like $\rho_{1 / 3}^{273}$; the ratio of centrifugal force to gravity $3 \Omega^{2} / 4 \pi G \rho \propto\left(\mathrm{H}^{2} / \mathrm{M}\right) \rho^{1 / 3}$, and so increases with decreasing scale. There is thus no analogue of the mass-flux relation. Overall contraction is ultimately halted in two dimensions, but if the cloud is cool it will spontaneously flatten along the rotation axis, so reducing $\Omega^{2} / G \rho$. If we temporarily ignore difficulties due to the shearing effect of nonuniform rotation, then we can expect sub-condensations to form, orbiting in centrifugal balance under the overall gravitational field of the cloud, but with the ratio of centrifugal force of spin to self-gravitation reduced below unity. (The fragmentation problem is somewhat 
relaxed: since centrifugal forces hold up the cloud, the dynamical problem is closer to the classical Jeans problem rather than the HoyleHunter problem, which demands that the blob density increase faster than that of the collapsing background). However, again isotropic contraction of each blob is limited by the growth of centrifugal forces of spin. The contrast with the magnetic case is clear. A modest degree of anisotropic flow can resolve the magnetic problem permanently, in the sense that subsequent isotropic contraction of a given mass $M$ is never halted, provided the condition $M>M_{(}(F)$ is satisfied. But in a rotating system in which sub-condensations ${ }^{c}$ are supposed to form without any removal of angular momentum of spin, there is always a limit to the density which a given mass can reach by isotropic contraction. A completely cold cloud could systematically flatten and fragment, but with a realistic value for the initial angular velocity the masses which could reach stellar densities would be far below stellar masses. And if we allow the gas to have a realistic temperature, then the hierarchical process just outlined would be halted by thermal pressure at densities much less than stellar order $-10^{-1} \mathrm{gm} / \mathrm{cm}^{3}$ or so.

The problem has been posed in its starkest form, with no transfer of spin angular momentum from a fragment to the surrounding medium. We can however be much more optimistic and suppose that some powerful braking process ensures that the spin of a contracting blob remains synchronous with that of the surrounding gas, at least for some stage of contraction and break-up. Then it is easy to see that with this assumption, the angular momentum problem could be resolved early in the evolution of the cloud. As an example, let the cloud have a density $\rho_{0} \simeq 30 \mathrm{~m}_{\mathrm{H}} / \mathrm{cm}^{3}$, and a mean angular velocity $\Omega \simeq 10^{-15}$, comparable with the local galactic orbital angular velocity, so that $3 \Omega_{0}^{2} / 4 \pi \mathrm{G} \rho_{0} \simeq 1$. Then suppose the cloud contracts isotropically, and at constant angular velocity, by a factor of no more than $1 / 30$, and then subsequently at constant angular momentum. The ratio $3 \Omega^{2} / 4 \pi \mathrm{G} \rho$ will not reach unity again until densities of $10^{18} \mathrm{~m}_{\mathrm{H}} / \mathrm{cm}^{3}$ (corresponding to the top of the Hayashi curve). The argument ${ }^{H}$ is easily generalised to apply to a hierarchical fragmentation picture, in which synchronism is maintained between a blob and its surroundings only for a limited degree of contraction, with further break-up occurring following flattening down the rotation axis. Some such process is envisaged in the scheme outlined by Bodenheimer (1976).

It is at this point that the presence of the magnetic field becomes an asset rather than a nuisance. First of all, if the field has a component perpendicular to the angular momentum vector, it will tend to iron out non-uniform rotation within the cloud. Secondly, field-lines linking a cloud with the surrounding galactic gas, or indeed a fragment with the rest of the cloud, can and will transport angular momentum. The absence of evidence for strong rotational velocities in dense molecular clouds may very well be due to magnetic braking during cloud formation. However, in making the optimistic estimate above it was assumed that braking process was so efficient that contraction occurred at constant angular velocity. If the cloud mass $M$ is less than the critical mass $M_{c}(F)$ for its flux, then indefinite contraction will in any 
case be prevented by the magnetic forces, so that the braking will proceed in its own time-scale, until corotation of cloud and surrounding gas has been reached. But if $M>M(F)$, then removal of angular momentum allows the cloud to contract further and so to spin up. (We again note the contrast with a rotating star, which is held up essentially by thermal pressure, with centrifugal forces a perturbation over the bulk; braking enables the star to become more nearly spherical, but will not lead to overall contraction). Thus if a cloud or fragment is to remain effectively co-rotating with the environment, braking must be so efficient that the characteristic time is short compared with the time of free-fall. But suppose instead that the time of braking is longer than the free-fall time. The cloud will then remain in approximate magneto-centrifugal-gravitational balance, and its rate of contraction will be determined not by gravity alone, but by the rate of braking. If $M$ were very close to $M_{C}(F)$, then the rotation rate would necessarily be kept well below its centrifugal balance value $\Omega \simeq(4 \pi G \rho / 3)^{\frac{2}{2}}$; but with $M$ markedly greater than $M_{C}(F),{ }_{1}$ force balance would be maintained only as long as $\Omega$ is close to $(4 \pi G \rho / 3)^{\frac{1}{2}}$, so that $\Omega$ would systematically increase as the cloud contracts. This in turn would mean that magnetic braking would be able to resolve the angular momentum problem completely, only if redistribution of angular momentum continues all the way to the pre-main-sequence phase.

It is thus clear that a reliable estimate for the rate of magnetic braking is of crucial importance. Ebert et al (1960) studied the case of a spherical cloud of radius $\bar{R}$ and density $\bar{\rho}$, rotating about an axis parallel to a uniform magnetic field $B_{0}$ linking the cloud with a surrounding medium of density $\rho_{0}$; the characteristic time found is $\tau_{b}=(8 / 5) \bar{R}\left(\rho / \rho_{\rho}\right)\left(1 / V_{A}\right)$,' where $V_{A}$ is the Alfvén speed in the external medium, a result which can be recovered by estimating the time for Alfven waves to trave 1 through a cylindrical $\mathrm{slab}$ with the same moment of inertia as the cloud. This time exceeds the free-fall time by a factor rather greater than $\left(\bar{\rho} / \rho_{0}\right)^{\frac{2}{2}}$. However, a more realistic treatment must allow for the distortion of the field as the contraction proceeds. Calculations still in progress (Gillis, Mestel and Paris 1974; 1976) adopt a field structure (symmetric about the rotation axis) that simulates the (undetached) field outlined in Section 3 . The results suggest that for clouds with $M$ greater than but close to $M_{c}(F)$, the time of braking may in fact be less than the free-fall time until the cloud has shrunk by a factor $\eta(F)<1$. If we consider a series of models with the flux decreasing steadily from the virial theorem maximum, the value of $\eta(F)$ increases: the lower the flux, the earlier the cloud changes over from a state of free-fall under negligible centrifugal forces, to a state of mechanical balance, with contraction determined by angular momentum loss.

A complete theory must synthesize all the different processes. When magnetic and angular momentum axes are more-or-less parallel, systematic fragmentation is possible via flow down the field-lines and magnetic redistribution of angular momentum (recall that three-dimensional contraction is required for the systematic break-up of an isothermal 
cloud). At some stage of contraction, the detachment of the field of a fragment from the background will inevitably cut off further angular momentum loss; however, the process can and will be repeated at the next stage of fragmentation. It can be plausibly argued that if fluxloss never becomes "fast" at any stage, so that the magnetic field is dynamically significant at all pre-opaque phases, then this combination of anisotropic flow and magnetic braking can yield break-up into a hierarchical distribution of proto-stars. Statistical energy interchange should tend in time to smooth out the spatial distribution within a cluster, but still leaving a sizeable proportion of binary and multiple sys tems.

The mathematical problems are more severe when there is a sizeable component of the magnetic field of a cloud perpendicular to the angular momentum axis. There is some evidence that magnetic braking will then be more efficient than in the parallel case (Kuls rud 1971; Mouschovias $1976 \mathrm{~d})$ though whether it can resolve the whole angular momentum problem early in the lifetime of the cloud again depends on the field's retaining an undetached structure for sufficiently long. Once the field has detached from the background, then a cloud with $B$ perpendicular to and with mass $M>M_{C}(F)$, and which has retained a sizeable angular momentum, can apparêntly achieve equilibrium as a roughly ellipsoidal body, with gravity balanced in one dimension by magnetic force, in another by centrifugal force, and in the third by magnetic and centrifugal forces jointly. No accurate models have yet been constructed, still less tested for stability. However, approximate studies (Mestel 1965) suggest that for a given value of $F / M$ there will be two equilibria. The model that is less prolate about the magnetic axis is unstable, and so can flatten along the magnetic axis and fragment. The more prolate model is prima facie stable: further slow loss of angular mgmentum would cause isotropic contraction, with $B$ increasing like $\rho^{2 / 3}$. Breakup can however occur after the cloud has lost flux via hydromagnetic instabilities (cf. Section 3 ); the ellipsoid would become more and more cylindrical about the field direction, and ultimately fragment e.g. into two self-gravitating blobs, orbiting in their mutual gravitational field. Again, contraction of each blob will be followed by magnetic braking of the spin. One can picture a new hierarchy, with each essential process - flux destruction, subsequent fragmentation, magnetic braking - having a characteristic time-scale comparable with the instantaneous free-fall time; provided flux-loss never becomes "fast" before the opaque phase is reached, so that the magnetic force remains comparable with the gravitational within each fragment, then again the scheme seems capable of producing systematic break-up of clouds into proto-stars.

If the magnetic axis remains essentially perpendicular to the rotation axis during all stages of contraction and break-up, we may expect a mass-spectrum more heavily biassed towards massive stars (cf. Section 7). However, the magnetic stresses on a rotating cloud or fragment will normally have a component perpendicular as well as parallel to the rotation axis: the field exerts a precessional as well 
as a braking torque, unless the field is either axially or equatorially symmetric with respect to the rotation axis. Paris (1971) showed that an oblique field coupling a fragment to a massive surrounding medium tends to rotate the angular momentum vector of the fragment towards parallelism with the field, though it was difficult to find a reliable time-scale estimate. It is worth noting here the observations by Markkanen (1976) of linear polarization of stars in the Pleiades cluster; he concludes that the rotation axis of $P$ leione is parallel to the direction of the field in the surrounding nebula.

\section{THE FLUX-FREEZING APPROXIMATION}

The scenarios outlined above assume that flux-loss from a fragment never becomes faster than the most rapid relevant dynamical process free-fall, braking, fragmentation. We have argued that the galactic magnetic field will not prevent fragmentation; in fact, so far from the field's being an embarrassment for star formation, we suggest that it is an asset, mainly because of the transfer of excess angular momentum and because of the suppression or control of rotational shear, and also as a possible extra parameter for fixing the final mass spectrum. We have noted that strict flux-freezing is a severe constraint, so that a gravitationally-distorted magnetic field is in fact likely at some stage to evolve into a lower energy state via local reconnection of field-lines. The new field structure may in turn be subject to dynamically-driven flux destruction, but the time-scale for this process is given by the Alfvén speed, which is at most of the order of the free-fall speed. Flux-freezing is relaxed sufficiently e.g. to allow the fragmentation of a prolate cloud with $B$ and $\Omega$ perpendicular, but the field remains "strong" at all stages, in the sense that the magnetic energy of a fragment is comparable with its gravitational energy.

In his original paper on fragmentation and the formation of Type II stars, Hoyle (1953) dealt with a primeval gas of atomic hydrogen which is kept at a temperature of near $10^{4}{ }^{0} \mathrm{~K}$ by collisional ionization followed by radiative cooling. The significant point is that the very processes by which compressional heat is radiated away imply a moderately high density of ions and electrons, so that any primeval magnetic field would inevitably remain frozen-in, in the sense that flux-loss never becomes "fast". However, in cool HI and molecular clouds, heat loss is primarily by processes unconnected with the degree of ionization. It is therefore not a priori obvious that the field remains strong throughout the whole epoch of formation of Type I stars. The question is important because as already noted, it is not yet clear whether the galactic magnetic field is likely to resolve the "angular momentum problem" in the early, low-density phases, when flux-freezing is certainly a good approximation. It may be that the magnetic stresses are able to keep the centrifugal forces of spin close to gravity at each stage of fragmentation, but cannot maintain for long effective corotation with the local galactic gas. If so, and if flux-loss does become rapid at some pre-proto-stellar phase, we shall need a different scenario to describe the later stages of star formation, with the magnetic field 
playing a much weaker role (cf. Section 6).

The time within which the electrons and ions (inductively coupled to the magnetic field) are forced out of a lightly ionized fragment of mass $M$ and containing flux $F$ is gi ven by $(9 \pi \alpha / 4)\left(n_{i} / n_{n}\right)(M / F)^{2}$, where $n_{i}, n_{n}$ are the densities of ions and neutral particles, and the frictional coupling constant $\alpha=$ (ion-neutral collision cross-section) (thermal velocity of neutrals)/m $\simeq 10^{15} \mathrm{cgs}$ in a cool cloud (Mestel and Spitzer 1956; Spitzer 1963; Mestel 1968; Nakano and Tademaru 1973; Nakano 1976; Silk 1976a). For a "magnetically strong" fragment with the flux near the virial theorem limit, this time reduces to $(5 \alpha / 4 \pi G)\left(n_{j} / n_{n}\right) \simeq 2 \times 10^{14}\left(n_{i} / n_{n}\right)$ years. Flux-loss becomes "rapid" if this time falls well below the time-scale of the relevant dynamical process - free-fall or angular momentum transfer. The crucial questions are (1) the rate of magnetic braking, and (2) the level to which $n_{j} / n_{n}$ falls. It is clear that flux-freezing remains an excellent approximation to much higher densities than in the first estimate (Mestel and Spitzer 1956), because of the higher collision cross-section (Osterbrock 1961), cosmic ray ionization (Hayakawa, Nishimura and Takay anagi 1961), radioactive ionization (Cameron 1962), and charge-transfer between molecular ions and metallic atoms (Oppenheimer and Dalgarno 1974). Nakano (1976) finds the ion density flattens off to $\simeq 10^{-1} / \mathrm{cm}^{3}$ when recombination on grains is included (without grain recombination $n_{i} / n_{n}$ would be larger by a factor $\simeq 10^{2}$ ). The flux-loss rate then equats the free-fall rate when $n_{n} \simeq 10^{10}$; however, if the fragment contracts in approximate centriługal balance, rapid flux-loss would occur at somewhat lower densities.

It should be noted that at $10^{10} / \mathrm{cm}^{3}$ a fragment is becoming optically thick (e.g. Low and Lynden-Bell 1976), and compressional heating at the free-fall rate would increase the temperature quasi-adiabatically: after a further density increase by a factor of $\simeq 10^{3}$, collisional ionization would begin to dominate. Thus it seems that if grain recombination occurs, and provided there is no local source of ionization, there is a narrow gap between the density at which flux-loss becomes rapid, and that at which thermal ionization takes over. However, there is another factor which merits detailed study. The processes of field reconnection and dynamical flux destruction may themselves yield locally generated cosmic rays. When flares occur on the sun we see a fraction of the magnetic energy released converted into high-speed particles. If similarly no more than one part in $10^{3}$ of magnetic energy destroyed during star formation goes into sub-cosmic ray particles, it could make a significant contribution to the ion density when $n \simeq 10^{9}-10^{10}$, and so extend still further the epoch over which flux destruction is restricted to the dynamical rate.

With so much theoretical uncertainty, one welcomes all the evidence that observation can provide on field-strengths in regions of different density. I would caution against the argument that rapid flux-loss must have occurred during the star formation phase, because of the comparative weakness of stellar magnetic fields. It is true that even the strongest 
fields observed in the magnetic Ap stars, when plausibly extrapolated inwards, yield magnetic fluxes much below the virial theorem limit. However, the relevant question is what would happen to a proto-star which managed to reach the pre-main sequence phase with a flux near this limit. We have already invoked dynamical instabilities to destroy excess flux during star formation, but emphasising that their time-scale is not shorter than the time of dynamical evolution of the cloud. But an opaque proto-star is by definition in approximate pressure equilibrium, and it contracts at a rate determined by heat loss - i.e. in the much longer Hayashi or Kelvin-Helmholtz time-scales rather than the free-fall time. Any instabilities will now have a much more leisurely time in which to develop. In particular, secular instabilities, dependent essentially on heat exchange, may cause flux-tubes to float to the surface in a time no longer than the overall contraction time - the "magnetic buoyancy" effect. It is even possible (cf. Levine 1974) that this destruction of a huge reservoir of magnetic energy may be connected with the $T$ Tauri phenomenon, as suggested by Lynden-Bell (this volume).

The argument should not be taken to imply that the "fossil" theory of stellar magnetism must be discarded. The origin of the "weak" fields observed in the strongly magnetic stars and surmised to be present deep down in other stars of both early and late type (including perhaps the sun) remains a matter for debate. They could still be a relic of the interstellar field from which the stars formed; but perhaps, more plausibly, they could be slowly decaying relics of fields built up by dynamo action during the Hayashi phase, after the interstellar field has all been destroyed (cf. Mestel 1976).

Even if flux-loss does become rapid during $s$ tar formation, this need not imply that magnetic effects can be subsequently neglected: the relic of the galactic field can still be important for angular momentum transport, albeit in a longer time-scale (cf. Section 6). Bisnovatyi-Kogan et al (1973) have explored the possibility that the turbulent velocities in clouds (or in proto-stars within clouds) could generate a magnetic field by the SKR dynamo mechanism. Their motivation is again to resolve the angular momentum problem; however, I suspect that in demanding that the braking should occur in a time short compared with the free-fall time, they are placing very severe constraints on the energetics of the dynamo process. If and when a star reaches the Hayashi phase, then a dynamo-built field is probable, and has available the much more leisurely thermal contraction time in which to remove more angular momentum, e.g. by coupling with a stellar wind.

\section{WEAKLY MAGNETIC, ROTATING SYSTEMS}

Once a fragment has become magnetically "weak", the Lorentz force cannot prevent substantial flattening along the rotation axis; and likewise no significant angular momentum redistribution can occur within a free-fall time. A field of highly supersonic turbulence could provide temporary support, but again the system will flatten as the turbulence decays. McCrea (1960, and this volume) has argued that the very process 
by which the turbulence deays - mutual collision and adherence of "floccules" moving randomly and with supersonic speeds - acts broadly like a gross turbulent viscosity, yielding a slowly rotating, condensed core containing most of the mass, surrounded by a Keplerian disk containing very little mass but most of the angular momentum - a protosolar system. Other authors (e.g. Ebert 1964; Cameron 1972) assume that the cloud flattens into a disk-like structure, without much angular momentum redistribution. The mass is concentrated towards the centre via the same gross gravitational instability that yields the flat rotation curve in disk-like galaxies (Mestel 1963; Hunter 1963). The problem then reduces to the evolution of such a "stellisk".

Ruzmaikina and Safranov (1976) have studied thermally-driven processes, analogous to the Eddington-Sweet circulation and the Goldreich-Schubert instabilities in stable stellar zones; they conclude that their effect on the angular momentum distribution is small. If the cloud retains a weak magnetic field with a component in the plane of the disk, then the twisting of the field by the non-uniform rotation will generate magnetic torques which will transport angular momentum and so also mass, since the gas will move so as to maintain near centrifugal balance (Mestel 1960). This may however also be less important than dynamical transport: the disk may be spontaneously unstable towards modes that transfer angular momentum outwards. In the galactic context, Lynden-Bell and Kalnajs (1972) associate the development of a gross spiral pattern with the tendency of a disk to develop internal gravitational torques. The resulting evolution time is long compared with a rotation period or a free-fall time, justifying the quasi-static assumption. Most workers explicitly or implicitly assume that disks will also be unstable on a smaller scale, so that a turbulent viscosity is continually maintained at the expense of the gravitational energy. The modes considered must be non-axisymmetric in order that azimuthal pressure gradients and gravitational forces break the constraint of detailed angular momentum conservation, so that Rayleigh's stability criterion is not violated. A self-gravitating locality of a thin disk is certainly gravitationally unstable; what remains unclear is whether a Keplerian disk, with negligible self-gravitation, is nevertheless unstable (e.g. von Weizsacker 1944, 1947). Attempts to prove instability have not carried conviction: Stewart (private communication) points out formal gaps in the analysis of all papers on this problem, including his own $(1975,1976)$. And even if turbulence does develop in a Keplerian disk, it is doubtful (ter Haar 1950) if much angular momentum could be transported from the central mass before the disk dissipates. This would seem to imply that the process must involve a substantial outflow of mass as well as angular momentum.

This scenario of turbulent or magnetic redistribution of disk angular momentum is currently being applied largely to $X$-ray sources. Lynden-Bell and Pringle (1974), who develop in mathematical detail the evolution of a viscous disk, suggest that the luminosity of a $T$ Tauri star is powered by the dissipation of a disk surrounding a star of solar mass. A more radical application is due to Gold $(1972,1974)$, 
who pictures a cold accumulation of matter, yielding first a cold degenerate central body of mass similar to Jupiter's. Jupiter has a metallic structure which strongly resists compression, so that the steady accumulation of more mass (again via viscous angular momentum transport) is accompanied initially by only a slight generation of heat, which is in any case rapidly conducted away. However, once the central mass has become of stellar order, the metallic structure breaks down, and the cold planet contracts towards a black dwarf state. Further mass accumulation will be accompanied by much more compressional heat generation, so that the black dwarf becomes a white dwarf of finite temperature: ultimately the hydrogen will ignite and the star will evolve from a degenerate into a non-degenerate structure, with a normal main sequence radius.

Gold argues further that his picture can account for the rotation rates of stars of different mass. An element of matter can reach the central body only when its angular momentum has been reduced to the value appropriate to the orbital velocity at the equator. When the white dwarf expands by a factor of 30 or more to become a normal star, the angular velocity decreases by $10^{-3}$, and the resulting main sequence star would necessarily be a slow rotator. Mass subsequently accreted by the same frictional process will have the specific angular momentum appropriate to the new radius, so that the angular velocity should increase with mass.

While some of these ideas have been foreshadowed (e.g. von Weizsacker 1944 , 1947), the picture with a degenerate central body is new and merits quantifying. Emphasis on the rate of compressional heating may not be relevant, as hydrogen fusion will begin even at zero temperature if the density is sufficiently high ("pycnonuclear reactions"). There is the danger that in evolving from a fully degenerate to a nondegenerate state, the star will reach temperatures (near $10^{8}{ }^{\circ} \mathrm{K}$ ) at which the thermonuclear fusion rate is so fast that the star will be destroyed (Mestel 1952). In the Gold picture the Hayashi phase never occurs, so some other evolutionary epoch must be associated with the $T$ Tauri phenomenon: a synthesis of the Gold and Lynden-Bell and Pringle pictures may be possible.

\section{THE LIMIT OF FRAGMENTATION AND THE INITIAL MASS SPECTRUM}

An essential part of Hoyle's scheme (1953) is that successive fragmentation ceases when the last fragments are sufficiently opaque for the heat of compression to be trapped rather than radiated away. Note that this criterion necessarily involves a characteristic time: more heat is generated during the free-fall of a slowly rotating fragment than during the agglomeration of mass at the centre of a disk via slow angular momentum transport. It is thus not clear that the simplest case - a non-rotating, non-magnetic cloud - will necessarily yield a lower bound to the mass-spectrum, even though the centrifugal and magnetic terms in the virial theorem act against gravity. However, it is certainly valuable to see what answer the simplest case yields to the 
minimum mass problem. A recent discussion by Rees (1976) puts the essence of the problem succinctly. A freely-falling fragment of mass $M$ and radius $r$ has the possibility of further fragmentation if it can radiate at something like the rate $\left(\mathrm{GM}^{2} / \mathrm{r}\right)(\mathrm{G} \rho)^{\frac{1}{2}}$. Now a cloud becomes opaque when its radiation rate per unit area approaches that of a black body: thus the condition that an opaque body should still be able to fragment implies essentially a lower limit on its temperature $T$. In standard notation the maximum radiation rate is $f a c \pi r^{2} T^{4}$, where $f(\leq 1)$ depends on the details of the physics and on the geometry of the fragment. Further fragmentation of an opaque body ceases to be possible when this maximum rate is inadequate to get rid of the compressional heat generated. Rees shows that this yjelds a limiting Jeans mas which can be written as $\left.M_{F} \simeq M_{c h} \mu^{-9 / 4} f^{-\frac{1}{2}}\left(k T / m_{p} c^{2}\right)^{\frac{1}{4}} \simeq 10-3 T / 10\right)^{\frac{19}{4}} f^{-\frac{5}{2}} \mu^{-9 / 4} M_{c h}$ where $\mathrm{M}_{\mathrm{ch}}=\left(\mathrm{Hc}_{\mathrm{c}} / \mathrm{Gm}^{2}{ }^{2}\right)^{3 / 2} \mathrm{~m}$ is approximately the Chandrasekhar mass. What immediately appears $p_{s}$ the remarkable insensitivity of this final fragment mass to the temperature - a variation from 10 to $10^{4}$ increases $M_{F}$ on ly by about 6 . There is little scope for variation in $\mu$; to increase $M_{F}$ above the very small value predicted above one must look for factors substantially reducing the efficiency factor $f$. Note that the density at which fragmentation ceases varies strongly with temperature $\left(\propto \mathrm{T}^{5 / 2}\right)$ : the higher the temperature the higher is the rate of compressional heat generation that the fragment can radiate away and so the smaller the radius which it can reach.

Rees's treatment has the merit of showing that a mass $M_{c h}$ of stellar order automatically appears. However, the factor f hides a lot of physics: both $f$ and $T$ depend on the track followed by contracting and fragmenting clouds in the $T-\rho$ plane, up to the point when they first become opaque, and so on their composition and environment. Low and Lynden-Bell (1976) study the details of the radiation transfer through an opaque spherical Jeans mass. They confirm that $M_{F}$ in a dark molecular cloud is small - $0.007 \mathrm{M}_{\odot}$ - and is insensitive to changes in the opacity. The minimum is reached at optical depth $\tau \simeq 3$ (justifying Rees's assumption of a small variation of $T$ through the fragment) and at densities $\simeq 5 \times 10^{11} / \mathrm{cm}^{3}$. Silk (1976a), using slightly different parameters, finds $M_{F} \simeq 0.01 M_{\odot}$.

One can hardly expect any fragmentation process to occur with complete efficiency, and it would not be surprising if some masses, large $r$ than these embarrassingly low values, do crash through the $\tau=3$ stage without having broken up (e.g. because of chance inelastic collisions between fragments). It is reasonable to ask for what mass range an opaque body at these densities can behave like a proto-star: i.e., settle into hydrostatic equilibrium with a large internal temperature gradient, and contract not at the free-fall rate, but at the slower rate determined by heat flow down the temperature gradient. It is known that a slight departure from hydrostatic balance will lead to a collapse rate close to free-fall; thus for hydrostatic balance to remain an excellent approximation, we require that the heat flow down the temperature gradient be less than the compressional heat that would be generated if the body were to contract at the free-fall rate. 
This procedure yields masses $\simeq 0.2 \mathrm{M}_{\odot}$ (Mestel and Spitzer 1956; Gaustad 1963). Proto-stars of smaller mass will contract Kelvin-Helmholtzwise until they reach internal temperatures of $\simeq 2000^{\circ} \mathrm{K}$, when dissociation and ionization of hydrogen leads to an effective $\gamma \simeq 1$ and a second collapse phase (Cameron 1962). Greater masses cannot retain enough heat to remain in hydrostatic equilibrium: they will collapse dynamically until dense and hot enough inside for gaseous rather than dust grain opacities to dominate.

Whether $0.007 \mathrm{M}_{\odot}$ or $0.2 \mathrm{M}_{\odot}$ is the typical mass to emerge from this simplest fragmentation mode $1^{\circ}$ is clearly linked with the details of the dynamics of fragmentation. Gaustad (1963) and Spitzer (1968) argue that masses $<0.2 \mathrm{M}$ will achieve a high degree of symmetry, and so will be less liable to fragment permanently during the second stage of collapse (due to hydrogen ionization); whereas larger masses, which never achieve an early Kelvin-Helmholtz phase, will be more likely to continue their break-up. Silk (1976a) suggests that the mass spectrum may indeed peak near $0.2 \mathrm{M}_{\theta}$, with special conditions being required to produce stars like the sun, let alone the minority of more massive stars.

One can hardly fail to agree with Rees that the stellar mass spectrum is likely to be controlled by the details of the dynamics of fragmentation. Rees's factor $f$ depends on geometry: Silk (1976a) and Hutchins (1976) point out that Hoyle's argument applied to a disk-like body yields $\simeq 0.1 \mathrm{M}_{\odot}$ rather than $0.007 \mathrm{M}_{\odot}$. Smith and Wright (1975) have emphasized the effect of heat from other fragments on thermal evolution. Silk (1976b) points out that radiation from the first-forming opaque fragments raises the temperature of the remaining gas, and so inhibits further fragmentation. He is thus able to derive an initial mass spectrum; however, this will presumably be affected by the occasional recombination of fragments following inelastic collisions (Layzer 1963; Arny and Weissman 1973).

My own feeling is that the angular momentum problem is paramount, and that resolution of the mass spectrum problem must await answers to the questions posed in Section 4-6. If the magnetic field becomes weak before the proto-stellar phase, and the angular momentum problem has not been fully resolved during star formation, then one of the scenarios of Section 6 would be relevant. But if the field remains "strong" all the way to the pre-main-sequence phase, then not only can it resolve the angular momentum problem but it must increase the mass of the fragments forming at each stage. We recall that if $B$ and $凡$ are more-or-less parallel, then fragmentation can occur following flow down the field-lines. Thermal pressure is all that limits break-up, and the final masses will again be fixed by the opacity: one hazards a guess that the centrifugal and magnetic forces will not increase $M_{F}$ by large factors. But if $\mathrm{B}$ and $\Omega$ are more-or-less perpendicular, then we noted that in some cases there is no obvious way in which the cloud can flatten along the field. Contraction occurs because of loss of angular momentum, but most of the gravitational energy is converted into 
magnetic and centrifugal energy rather than into the random kinetic energy that is the basic requirement of fragmentation. Break-up into mutually orbiting blobs can occur, but only following dynamical flux destruction, and this requires a sizeable gravitational distortion of the field. If the magnetic field remains "strong" all the way to the proto-star phase, then the inhibition of fragmentation could lead to final masses far above the $0.007 \mathrm{M}_{\odot}$ or $0.2 \mathrm{M}_{\odot}$ estimates discussed above.

A final remark on the build-up of massive $O$ and $B$ stars by accretion of interstellar gas by stars of lower mass. A weakness of the earlier work (McCrea 1953; Mestel 1954) is the implicit requirement of a comparatively low angular momentum of the inflowing gas about the star. If however one postulates that the star has some magnetic flux with some field-lines emerging from the surface, this objection may be removed. Inflowing gas of low density will be forced to corotate with the star, the excess angular momentum being transferred inwards by the magnetic stresses: this could simultaneously ensure that these stars rotate rapidly.

\section{REFE RENCES}

Arny, T. and Weissman, P. 1973, Astronom. J. 78, 309.

Bisnovatyi-Kogan, G.S., Ruzmaikin, A.A. and Sunyaev, R.A. 1973, Astron. J.USSR $50,210$.

Bodenheimer, P. 1976, (private communication).

Bonnor, W.B. 1956 , Mon.Not.R.Astr.Soc. 116, 351.

Cameron, A.G.W. 1962, Icarus 1, 13.

Came ron, A.G.W. 1972, in On the Origin of the Solar System, ed.H. Reeves (C.N.R.S., Paris).

Chandrasekhar, S. and Fermi, E. 1953, Astrophys.J. 118, 116.

Ebert, R. 1955, Z.f.Astrophys. 37, 217.

Ebert, R. 1964, Habilitationschrift, Un.Frankfurt-am-Main .

Ebert, R., von Hoerner, S. and Temesvary, S. 1960, Die Entstehung von Sternen (Springer-Verlag, Berlin).

Garlick, A.R. 1976, (preprint).

Gaustad, J.E. 1963, Astrophys.J. 138, 1050.

Gillis, J., Meste1, L. and Paris, R.B. 1974, Astrophys.Sp.Sci. 27, 167.

Gillis, J., Meste1, L. and Paris, R.B. 1976 (in preparation).

Gold, T. 1972, High Energy Activity During the Late Phases of Stellar Evolution, Accademia dei Lincei, Rome.

Gold, T. 1974, Phil.Trans.R.Soc.Lond. A $277,453$.

Hay akawa, S., Nishimura, S. and Takay anagi, K. 1961, Pub.Astr.Soc. Japan 13, 184.

Hoyle, F. 1953, Astrophys.J. 118, 513.

Hunter, C. 1962, Astrophys.J. 135, 594.

Hunter, C. 1963, Mon.Not.R.Astr.Soc. 126, 299.

Hunter, C. 1964, Astrophys.J. 139, 570.

Hutchins, J.B. 1976, Astrophys. J. 205, 103.

Kuls rud, R.M. 1971, Astrophys.J. 163, 567.

Layzer, D. 1963, As trophys.J. 137, 351.

Levine, R.H. 1974, Astrophys.J. 190, 457. 
Lin, C.C., Meste1, L. and Shu, F.H. 1965, Astrophys.J. 142, 1431.

Low, C. and Lynden-Be11, D. 1976, Mon.Not.R.Astr.Soc. 176, 367.

Lynden-Be11, D. 1973, in G. Contopoulos, M. Hénon, D. Lynden-Be11, Dynamical Structure and Evolution of Stellar Systems, Saas-Fee Lectures.

Lynden-Be11, D. and Kalnajs, A.J. 1972, Mon.Not.R.Astr.Soc. 157, 1. Lynden-Be11, D. and Pringle, J.E. 1974, Mon.Not.R.Astr.Soc. 168, 603. McCrea, W.H. 1953, Mon.Not.R.Astr.Soc. 113, 162. McCrea, W.H. 1957, Mon.Not.R.Astr.Soc. 117, 562. McCrea, W.H. 1960, Proc.Roy.Soc. A 256, 245.

Markey, P. and Tayler, R.J. 1973, Mon.Not.R.Astr.Soc. 163, 77. Markkanen, T. 1976 (preprint).

Meste1, L. 1952, Mon.Not.R.Astr.Soc. 112,583 and 598.

Meste1, L. 1954, Mon.Not.R.Astr.Soc. 114, 437.

Meste1, L. 1960, Vistas in Astronomy 3, 296.

Mestel, L. 1963, Mon.Not.R.Astr.Soc. $126,553$.

Meste 1, L. 1965, Quart.J.R.Astr.Soc. 6, 161 and 265.

Meste1, L. 1966, Mon.Not.R.Astr.Soc. 133, 265.

Mestel, L. 1969, in Plasma Instabilities in Astrophysics, ed. D.A.Tidman and D.G. Wentzel (Gordon and Breach, New York).

Mestel, L. 1976, IAU Colloquium No.32, Physics of Ap Stars, ed.

W. W. Weiss (D. Reidel, Ams terdam).

Meste 1, L. and Paris, R.B. 1976, (in preparation).

Meste 1, L. and Spitzer Jr., L. 1956, Mon.Not.R.Astr.Soc. 116, 583.

Meste1, L. and Strittmatter, P.A. 1967, Mon.Not.R.Astr.Soc. 137, 95.

Mouschovias, T.Ch. 1976a, Astrophys.J. 206, 753.

Mouschovias, T.Ch. 1976b, Astrophys.J. 207, 141.

Mouschovias, T.Ch. $1976 \mathrm{c}$, Astrophys.J. (in press).

Mouschovias, T.Ch. $1976 \mathrm{~d}$, (in preparation).

Nakano, T. 1976, Publ.Astron.Soc.Japan 28, 355.

Nakano, T. and Tademaru, E. 1972, Astrophys.J. 173, 87.

Oppenheimer, M. and Dalgarno, A. 1974, Astrophys.J. 192, 29.

Paris, R.B. 1971, PhD. dissertation, Manchester University.

Parker, D.A. 1973, Mon.Not.R.Astr.Soc. 163, 41.

Parker, D.A. 1974, Mon.Not.R.Astr.Soc. $168,331$.

Rees, M.J. 1976, Mon.Not.R.Astr.Soc. 176, 483.

Ruzmaikina, T.V. and Safronov, V.S. 1976, Astron.J.USSR. 53, 860.

Silk, J. 1976a,b, Astrophys.J. (in press).

Smith, R.C. and Wright, A.E. 1975, Mon.Not.R.Astr.Soc. 172, 221.

Spitzer Jr., L. 1963, in Origin of the Solar System, ed. R.Jastrow and A.G.W.Cameron (Academic Press, New York).

Spitzer Jr., L. 1968, in Nebulae and Interstellar Matter, ed.

B. M. Middlehurst and L. H. Aller (University of Chicago Press, Chicago).

Stewart, J.M. 1975, Astron. Astrophys. 42, 95.

Stewart, J.M. 1976, Astron. Astrophys. 49, 39.

Strittmatter, P.A. 1966, Mon.Not.R.Astr.Soc. $132,359$.

ter Haar, D. 1950, Astrophys.J. 111, 179.

Verschuur, G.L. 1969, in Plasma Instabilities in Astrophysics, ed. D. A. Tidman and D. G. Wentzel (Gordon and Breach, New York). 
von Weizsăcker, C.F. 1944, Z.f.Astrophys. 22, 319.

von Weizsăcker, C.F. 1947, Z.f.Astrophys. 24, 181.

Vrba, F.J. 1976, PhD thesis, Un. of Arizona.

Vrba, F.J., Strom, S.E. and Strom, K.M. 1976, (preprint).

Wright, G.A.E. 1973, Mon.Not.R.Astr.Soc. $162,359$. 